\title{
The $Z_{6}$-symmetric model partition function on triangular lattice
}

\author{
Nor Sakinah Mohd Manshur, Siti Fatimah Zakaria*, Nasir Ganikhodjaev
}

Department of Computational and Theoretical Science, Kulliyyah of Science, International Islamic University Malaysia, Kuantan Campus, 25200 Kuantan, Pahang, Malaysia

* Corresponding author: fatimahsfz@iium.edu.my

\section{Article history}

Received 17 August 2019

Revised 15 January 2020

Accepted 28 January 2020

Published Online 15 June 2020

Graphical abstract

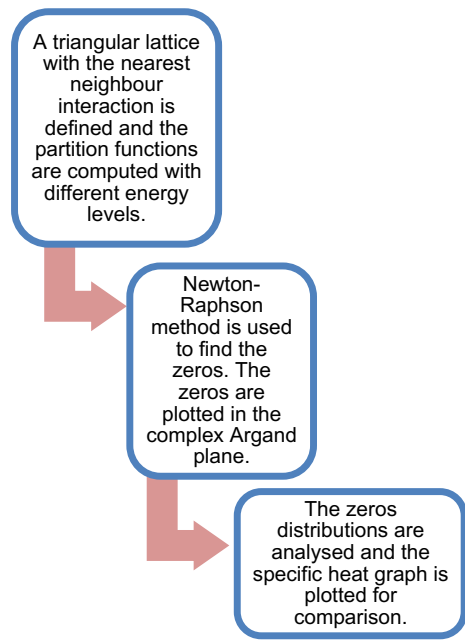

\begin{abstract}
There is a study on a square lattice that can predict the existence of multiple phase transitions on a complex plane. We extend the study on the different types of $Z_{Q}$-symmetric model and different lattices in order to provide more evidence to the existence of multiple phase transitions. We focus on the $Z_{Q}$-symmetric model with the nearest neighbour interaction on the six spin directions between molecular dipole, i.e. $Q=6$ on a triangular lattice. Mainly, the model is defined on the triangular lattice graph with the nearest neighbour interaction. By using the transfer matrix approach, the partition functions are computed for increasing lattice sizes. The roots of polynomial partition function are also computed and plotted in the complex Argand plane. The specific heat equation is used for further comparison. The result supports the existence of the multiple phase transitions by the emergence of the multiple line curves in the locus of zeros distribution for specific type of energy level.
\end{abstract}

Keywords: Statistical mechanics, $Z_{Q}$-symmetric model, partition function, triangular lattice, phase transition

\section{INTRODUCTION}

We study a complex system of mathematical model in statistical mechanics [1]. The study is inspired from the case of well-known Ising model [2] of 2 spin states to the case with more than 2 spin states that is $Q$-state Potts model of a physical system. The $Q$ in both $Q$-state Potts and $Z_{Q^{-}}$-symmetric models is the number of possible spin directions of a physical state. In contrast to the $Q$-state Potts model, our model also considers the interaction between nearest neighbour spin states but with additional relation to angle of the spin directions. The energy from the interaction is depends on this assumption.

Our interest here is for $Q=6$ (the case $Q=5$ has been studied and discussed in separate paper [3]). In $Z_{Q^{-}}$-symmetric model, the $Q=2$ and 4 are related to Ising model and $Q=3$ is related to 3 -state Potts model [1]. One of the most celebrated results for phase transition is discussed in work by Onsager and his student Kaufmann $[4,5,6]$. They described the exact solution for the Ising model on square lattice.

The study of zeros of partition function was first discussed by Fisher [7] on the Ising model on square lattice, where the loci of 2 circles were found in the distribution. One of the circles was cut at the real axis at exactly the phase transition point in the ferromagnetic region. This study on zeros distribution was also done in many other cases for the Ising model and the $Q$-state Potts model, for example work by $[1,8,910,11,12]$. For the $Z_{Q}$-symmetric model, Martin [1] and Zakaria [8] suggested that the existence of the multiple phase transitions could be predicted by the emergence of the multiple linear curves on the complex temperature plane.

In this study, we extend our previously studied $Z_{5}$-symmetric model [3] in order to put more evidence regarding the existence of the multiple phase transitions of the $Z_{Q}$-symmetric model on triangular lattice. We study specifically for the partition function of the $Z_{6^{-}}$ symmetric model on triangular lattice.

We initially present the definitions of the graph and the $Z_{Q^{-}}$ symmetric model. The Hamiltonian on the triangular lattice is defined and its function is computed for several cases of energy level. The partition functions of these many cases of energy level are computed in increasing lattice sizes. The zeros of the partition functions are computed and their results are analysed in the complex Argand plane. Finally, we compute and plot the specific heat equation for comparison.

\section{Graph}

The magnetic dipole moments of atomic spins are represented by discrete variables. The graph represents the spin particles on a lattice where each spin interacts with its nearest neighbours.

Definition 1 [13] A directed graph $\Lambda$ is a triple $\Lambda=(V, E, f)$. The $V$ is a set. The elements $v \in V$ are called vertices. The $E$ is also a set. The elements $e \in E$ are called edges. The $f$ is a function $f: E \rightarrow$ $V \times V$. Given $e \in E$ and $v_{1}, v_{2} \in V$, the images $f(e)=\left\langle v_{1}, v_{2}\right\rangle$ give the 'source' and 'target' vertex of edge e. 
Definition 2 [8] The distance $d_{\Lambda}(u, v)$ is the number of edges in the shortest path from $u$ to $v$. Two vertices $u, v \in V$ are called nearest neighbours if $f(e)=\left\langle v_{1}, v_{2}>\right.$ for some $e \in E$ i.e. when $d_{\Lambda}(u, v)=$ 1.

Definition 3 [3] Consider a set $\Lambda$ of lattice sites, a $d$-dimensional lattice is formed from each site of the nearest neighbours. There is a discrete variable $\sigma_{k}$ such that $\sigma_{k} \in\{1,2, \ldots, Q\}$ for each lattice site $k \in \Lambda$ which representing the site's spin. A spin configuration, $\sigma=\left(\sigma_{k}\right)_{k \in \Lambda}$ is an assignment of spin value to each lattice site.

\section{The triangular lattice}

We consider the triangular lattice (a connection of 3 edges formed a triangle shape - see [6] for different examples and shapes) with the nearest neighbour interaction between molecular dipole in increasing lattice sizes.

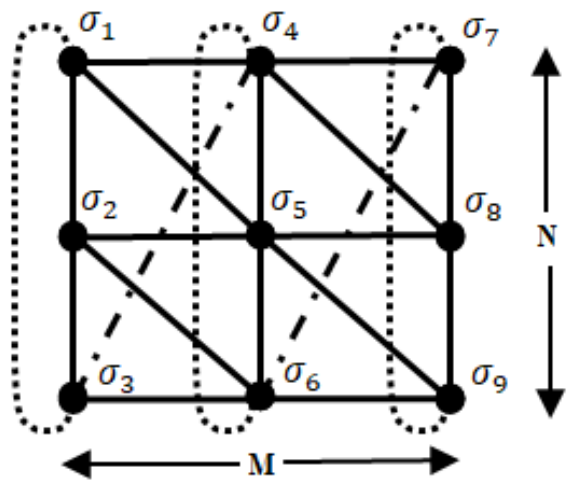

Figure 1 Triangular lattice with system size $\mathrm{N} \times \mathrm{M}^{\prime}=3 \times 3^{\prime}$.

Figure 1 shows 3 by 3 triangular lattice with $\mathrm{N}$ and $\mathrm{M}$ represent the number of row vertices and column vertices, respectively. The dash dot lines and the dot lines correspond to the edges that connecting the upper and lower vertices, giving a cylindrical triangular lattice. This cylindrical shape corresponds to the periodic boundary condition. The symbol prime in $\mathrm{M}^{\prime}$ shows that it is an open boundary condition whereas the number without prime indicates that it is periodic boundary condition.

We study the periodic boundary condition for the vertical direction and the open boundary condition for the horizontal direction.

\section{METHODS}

The $Z_{Q}$-symmetric model's energy relation is represented by the idea of a clock-like circle. The energy is depended on the interaction between one spin to another. The difference of the energy is always the same when any pair of the direction is moved with a fixed angle around the clock. This angle is associated to the symmetry of the model [14].

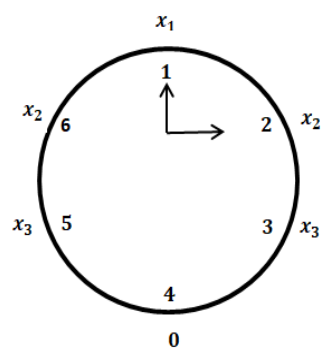

Figure 2 The spin directions of one spin relative to spin 1 represented by a pair of arrow.

Figure 2 shows the example of the interaction of two spin directions represented by two arrows relative to spin 1 . The numbering inside the clock corresponds to the spin direction while outside the clock corresponds to the energy.

We define the Hamiltonian function of the $Z_{Q}$-symmetric model as follows. Let $\sigma \in \Omega$.
Definition 4 The Hamiltonian of the $Z_{Q}$-symmetric model is defined as

$$
\begin{aligned}
H_{\chi}(\sigma)= & -J \sum_{\substack{\langle i, j\rangle=f(e) \\
e \in E}} \chi\left[\sigma_{i}-\sigma_{j}\right] \\
=-J & \sum_{\substack{\langle i, j\rangle=f(e) \\
e \in E}} \sum_{r=1}^{\left[\frac{Q}{2}\right]} \gamma_{r} \cos \left(\frac{2 \pi r\left(\sigma_{i}-\sigma_{j}\right)}{Q}\right) \\
& +\tilde{\gamma}_{r}
\end{aligned}
$$

where $\left[\frac{Q}{2}\right]$ is the discrete value of the division and $\gamma_{r}, \tilde{\gamma}_{r} \in \mathbb{R}$ are model parameters fixed for a given model.

The Hamiltonian depends on the angle between two spins. A small angle will contribute to lesser energy loss as compared to a large angle. The energy list is written as $\chi=\left(x_{1}, x_{2}, x_{3}, 0\right)$. And its energy loss is called an energy penalty, that is given by the absolute value of energy differences, $\left(\left|x_{2}-x_{1}\right|,\left|x_{3}-x_{2}\right|,\left|x_{4}-x_{3}\right|\right)$.

\section{Partition function and transfer matrix approach}

Definition 5 For a given $Q$ and $\Lambda$, the partition function is defined as

$$
\mathrm{Z}_{\Lambda}(\beta)=\sum_{\sigma \in \Omega_{\Lambda}} \exp \left(-\beta \mathcal{H}_{\Lambda}(\sigma)\right) ;
$$

where $\beta=1 /\left(k_{B} T\right)$ in which $k_{B}$ refers to the Boltzmann's constant and $T$ refers to the absolute temperature.

The partition function is a function that relates temperature and other parameters with the states of a spin system $[15,16]$, which allows the energy transfer to its neighbour and environment.

The partition function of $Z_{6}$-symmetric model, $Z(x)$ is computed by transfer matrix approach $[1,8,15]$

Definition 6 Let $G, G^{\prime}$ be two lattice graphs. Let $E$ be the set of edges and $V$ be the set of vertices. For the union of two graphs $G \cup G^{\prime}$ we have

and

$$
V_{G \cup G^{\prime}}=V_{G} \cup V_{G^{\prime}}
$$

where $E_{G} \cap E_{G^{\prime}}=\emptyset$.

$$
E_{G \cup G^{\prime}}=E_{G} \cup E_{G^{\prime}},
$$

Based on the Chapman-Kolmogorov theorem [17], the summation of the product of partition vectors for graph $\Lambda$ and $\Lambda^{\prime}$ can produce the partition function of a new combined graph $\Lambda \Lambda^{\prime}$.

The partition vector is reorganised into a matrix called a transfer matrix. Let $T$ be a transfer matrix. Each entry in matrix $T$ is given by

$$
T_{i j}=\left.Z_{\Lambda}^{V}\right|_{\sigma_{i} \in \Omega_{V_{I}}, \sigma_{j} \in \Omega_{V_{O}}}
$$

in which $V_{I}$ represents the incoming site and $V_{O}$ represents the outgoing site such that $V=V_{I} \cup V_{O}$. The row matrices correspond to the set of all possible configuration states $\Omega_{V_{I}}$ while the column matrices correspond to the set of all possible configuration states $\Omega_{V_{O}}$. See Figure 3.

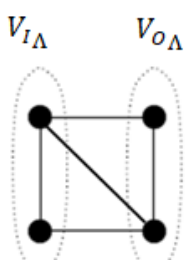

$\Lambda$

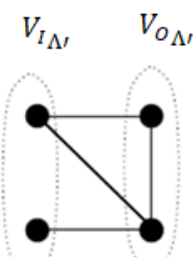

$\Lambda^{\prime}$

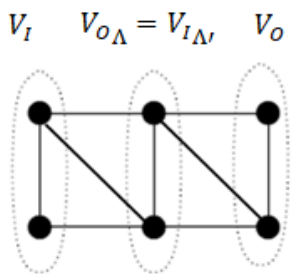

$\Lambda \Lambda^{\prime}$
Figure $3 \mathrm{~A}$ new lattice graph $\Lambda \Lambda^{\prime}$ is formed from the combination of graphs $\Lambda$ and $\Lambda^{\prime}$. 
The dot circles in Figure 3 correspond to incoming and outgoing spins which denoted as $V_{I_{\Lambda}}$ and $V_{O_{\Lambda}}$ respectively for $\Lambda$, and $V_{I_{\Lambda^{\prime}}}$ and $V_{O_{\Lambda^{\prime}}}$ respectively for $\Lambda^{\prime}$. A new graph $\Lambda \Lambda^{\prime}$ is formed when the graphs $\Lambda$ and $\Lambda^{\prime}$ are connected.

Based on equation (3) and Chapman-Kolmogorov Theorem [17], the transfer matrix multiplication can be used to combine two graphs where the function is given by,

$$
\mathrm{T}_{\Lambda \Lambda^{\prime}}=\mathrm{T}_{\Lambda} \mathrm{T}_{\Lambda^{\prime}}
$$

where $V_{I_{\Lambda \Lambda^{\prime}}}=V_{I_{\Lambda}}$ and $V_{O_{\Lambda \Lambda^{\prime}}}=V_{O_{\Lambda^{\prime}}}$.

Then, the new partition function is given by

$$
Z_{\Lambda \Lambda^{\prime}}=\sum_{i=1}^{d} \sum_{j=1}^{d}\left(\mathrm{~T}_{\Lambda \Lambda^{\prime}}\right)_{i, j}
$$

where $i, j$ is the index of matrix $T_{\Lambda \Lambda^{\prime}}$ and $d$ is the dimension of the matrix.

\section{Computation of the zeros}

The Newton-Raphson method and a $\mathrm{C}++$ programming language are used for computation in finding the zeros of the partition function (with the Gnu multiple precision library as another essential tool). Then, the zeros are plotted in the complex Argand plane.

\section{RESULTS AND DISCUSSION}

\section{Zeros distribution}

The results of the zeros distribution of the partition function are presented in this section. The zeros are plotted in the complextemperature Argand plane.

The zeros of the partition functions are studied for some energy list $\chi$ in increasing lattice sizes. The largest possible triangular lattice (due to limitation of our available computing resources) is size 8 by 8 .

The ferromagnetic region is enclosed by the real part $[1, \infty)$ with $J>0$ while the antiferromagnteic region is enclosed by the real part $[0,1]$ with $J<0$. The other region is unphysical.

We list the considered cases which give results of the zeros distribution of the partition function for $Z_{6}$-symmetric model in Table

Table 1 The zeros distribution computed cases with

\begin{tabular}{|c|c|c|c|c|c|}
\hline & $\chi$ & $5 \times 5$ & $6 \times 6^{\prime}$ & $7 \times 7^{\prime}$ & $8 \times 8^{\prime}$ \\
\hline \multirow{5}{*}{$Q=6$} & $(2,1,0,0)$ & $\checkmark$ & $v$ & $\checkmark$ & $\checkmark$ \\
\hline & $(2,1,1,0)$ & $\checkmark$ & $v$ & $\checkmark$ & $\checkmark$ \\
\hline & $(3,1,0,0)$ & $\checkmark$ & v & $\checkmark$ & $\checkmark$ \\
\hline & $(3,2,0,0)$ & $\checkmark$ & v & $\checkmark$ & $\checkmark$ \\
\hline & $(3,2,1,0)$ & $\checkmark$ & $v$ & $\checkmark$ & $\checkmark$ \\
\hline
\end{tabular}
specific energy list $\chi=\left(x_{1}, x_{2}, x_{3}, 0\right)$.

The zeros distributions for several cases of size 8 by 8 triangular lattice are presented in Figures 4 through 8.

Figure 4 shows the zeros distribution for energy level $\chi=(2,1,0,0)$. As the size increases, the zeros in the positive real axis form some linear arcs. The arcs of zeros are approaching the real axis in both ferromagnetic and antiferromagnetic regions. We can see some zeros outside the linear arc in ferromagnetic part. It may be due to the lattice size and boundary effects, but may be also forming another linear arc. This observation will be clearer if we can compute for bigger cases.

The zeros distribution for $\chi=(2,1,1,0), \mathrm{N}=7$ and 8 are shown in Figure 5. The appearance of the arcs is clear as the lattice size increases. There are branches of zeros that approaching the origin. In antiferromagnetic region, the existence of phase transition behaviour is not clear since we do not have enough zeros in this region.

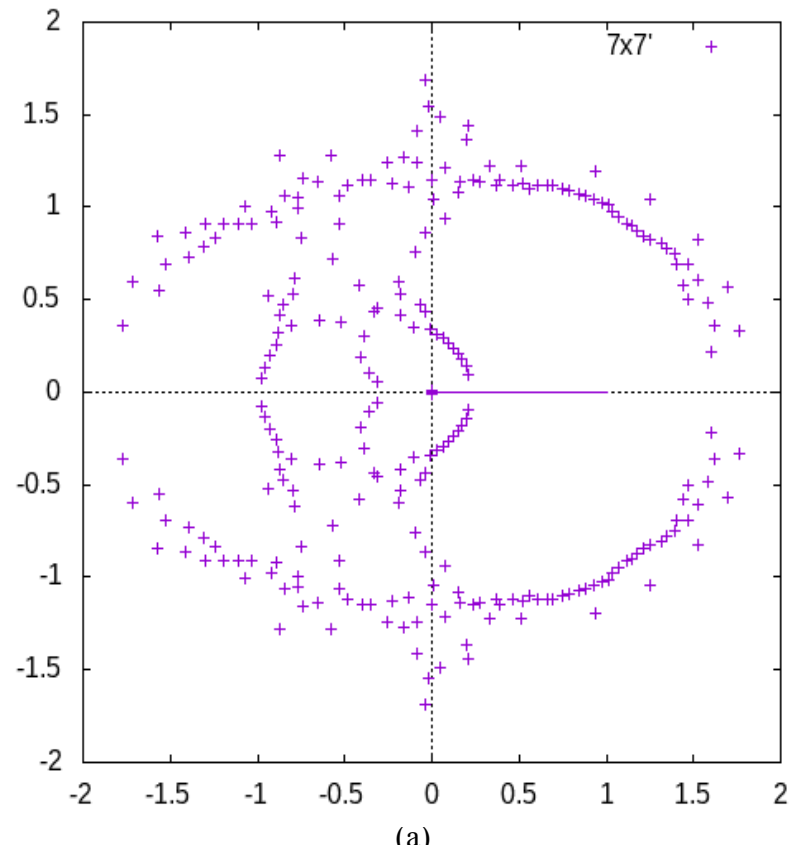

(a)

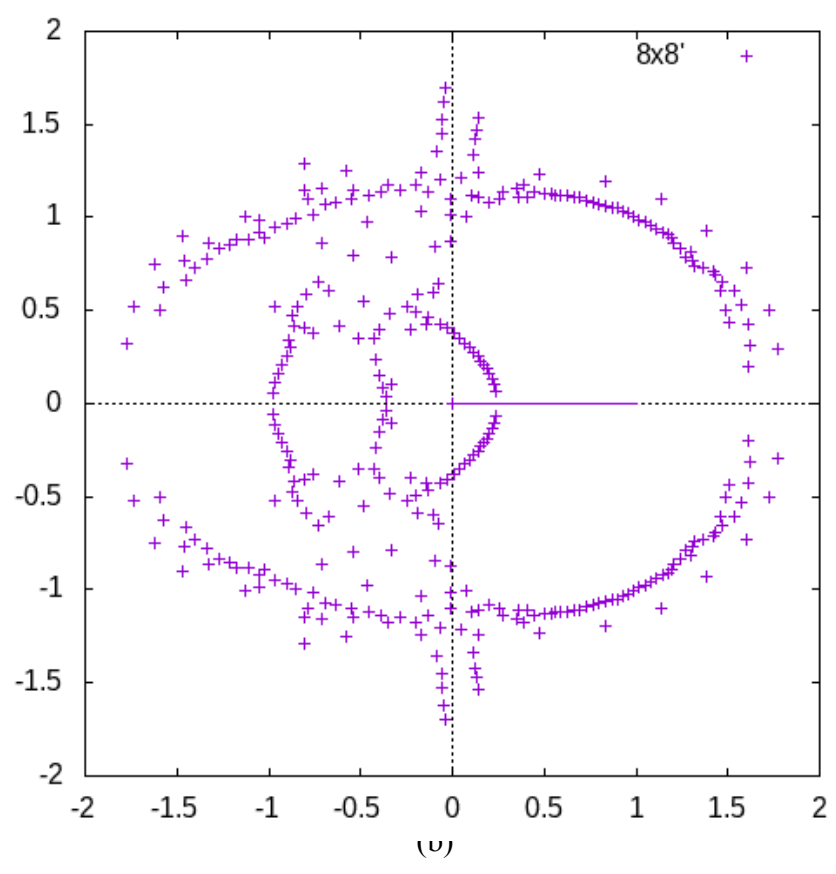

Figure 4 Zeros distribution for $\chi=(2,1,0,0)$; a) $\mathrm{N}=7, \mathrm{~b}) \mathrm{N}=8$.

We present the zeros distribution for $\chi=(3,1,0,0)$ in Figure 6 . The arcs of zeros are approaching the real axis in both ferromagnetic and antiferromagnetic regions. There is a branch of zeros in non-physical region that is moving closer to the real axis as the lattice sizes increases. We can also clearly see that the graph of the zeros distribution in $\mathrm{N}=8$ lattice size gives a better illustration as compared to the smaller lattice size, especially in antiferromagnetic part. The zeros are distributed in line.

We continue to further study the zeros distribution for $\chi=(3,2,0,0)$, as referred to Figure 7. At this energy level, the zeros distribution is more complicated as compared to the previous energy levels. In both ferromagnetic and antiferromagnetic regions, as the lattice size increases, we can see that there are multiple arcs of zeros in the ferromagnetic region but it is not very clear either the zeros will merge into one line or remain in multiple lines for larger lattice sizes. In the non-physical region, some branches of zeros are moving towards the real axis. 


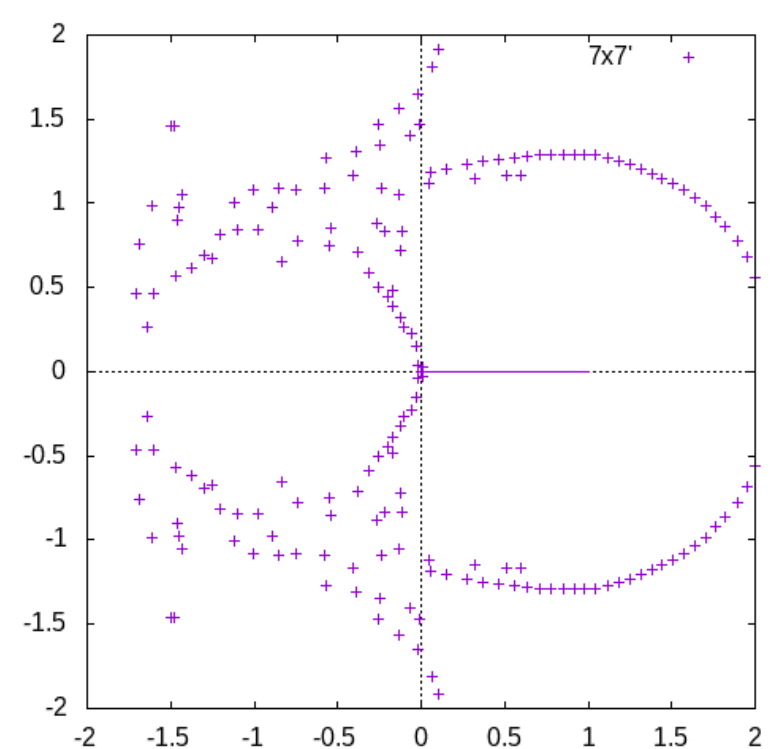

(a)

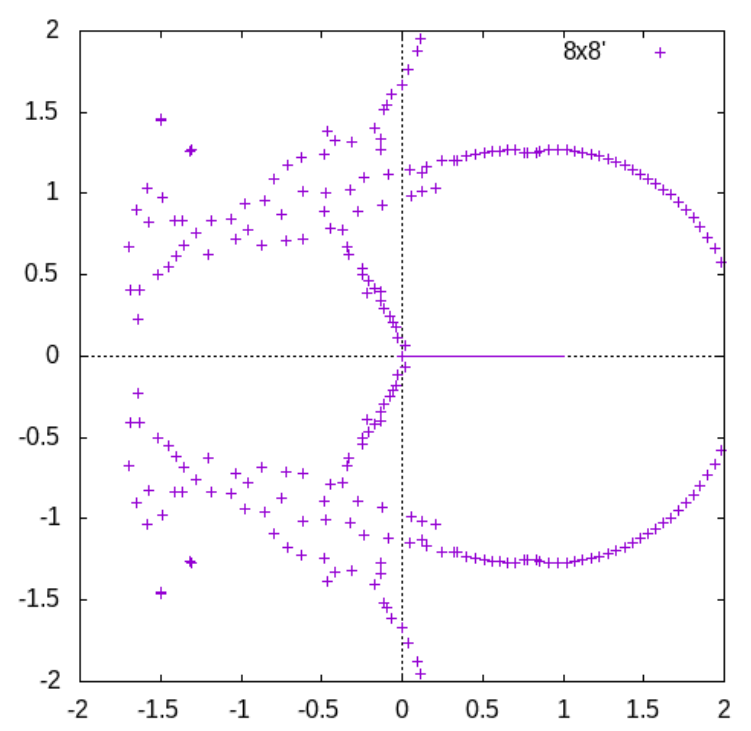

(b)

Figure 5 Zeros distribution for $\chi=(2,1,1,0)$; a) $\mathrm{N}=7, \mathrm{~b}) \mathrm{N}=8$.

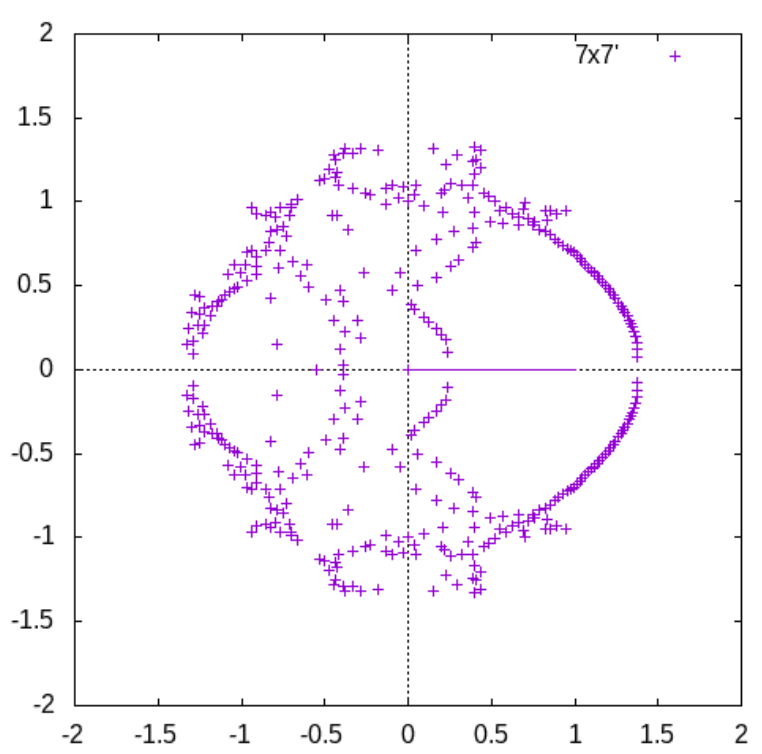

(a)

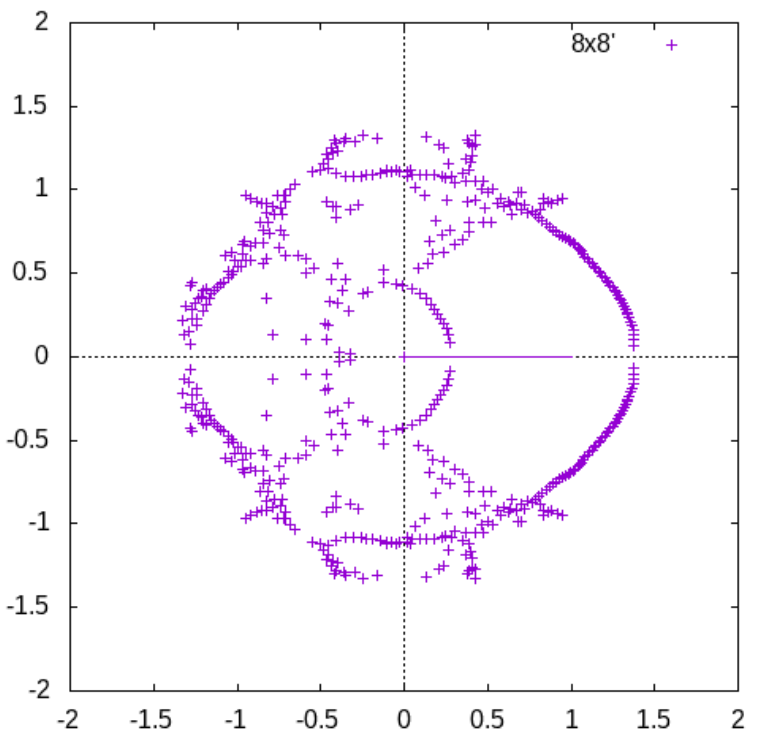

(b)

Figure 6 Zeros distribution for $\chi=(3,1,0,0)$; a) $N=7$, b) $N=8$.

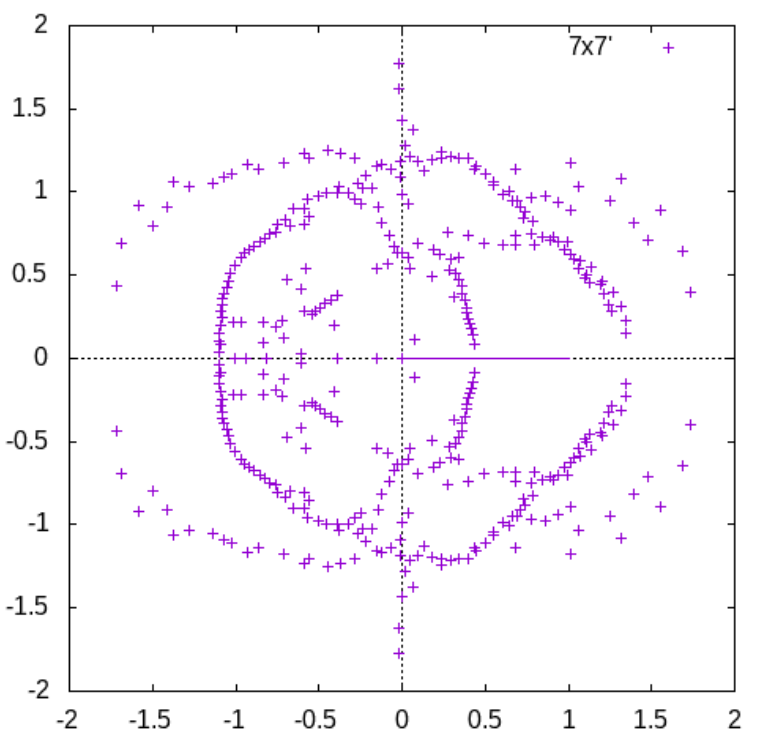

(a)

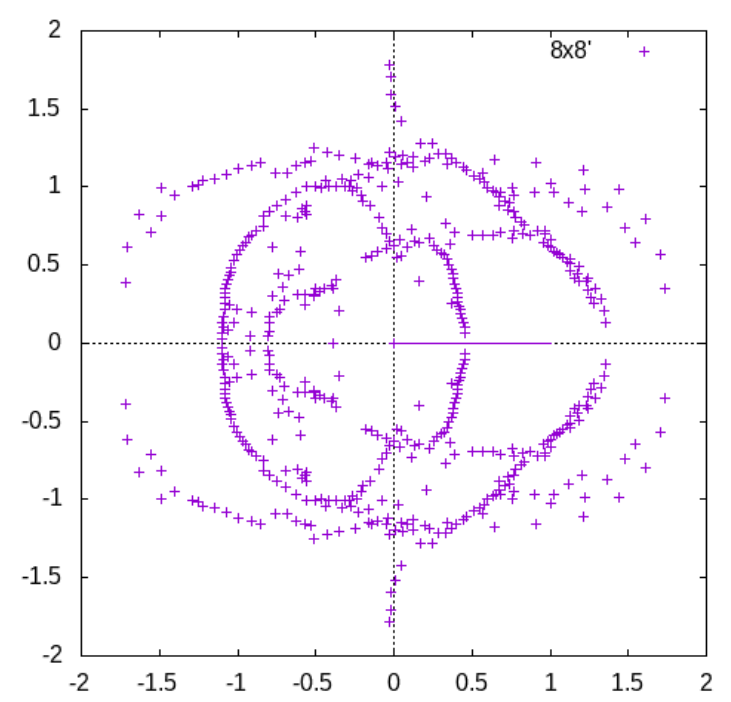

(b)

Figure 7 Zeros distribution for $\chi=(3,2,0,0)$; $a) \mathrm{N}=7, \mathrm{~b}) \mathrm{N}=8$. 
Lastly, as seen in Figure 8 for $\chi=(3,2,1,0)$, we observe that the zeros have a unique distribution as compared to the previous energy levels. Here, we have symmetry (left and right) in the zeros distribution. Other cases that we have covered do not have this symmetry. This $\chi=$ $(3,2,1,0)$ is the only case we considered that has energy penalty that is fairly distributed $(1,1,1)$ - one additional value of energy is lost for each addition of a fixed angle. This characterises the partition function to exhibit a symmetry. In the ferromagnetic region, the arcs of zeros are approaching the real axis but it is not so clear to determine either it has one single curve or multiple curves.

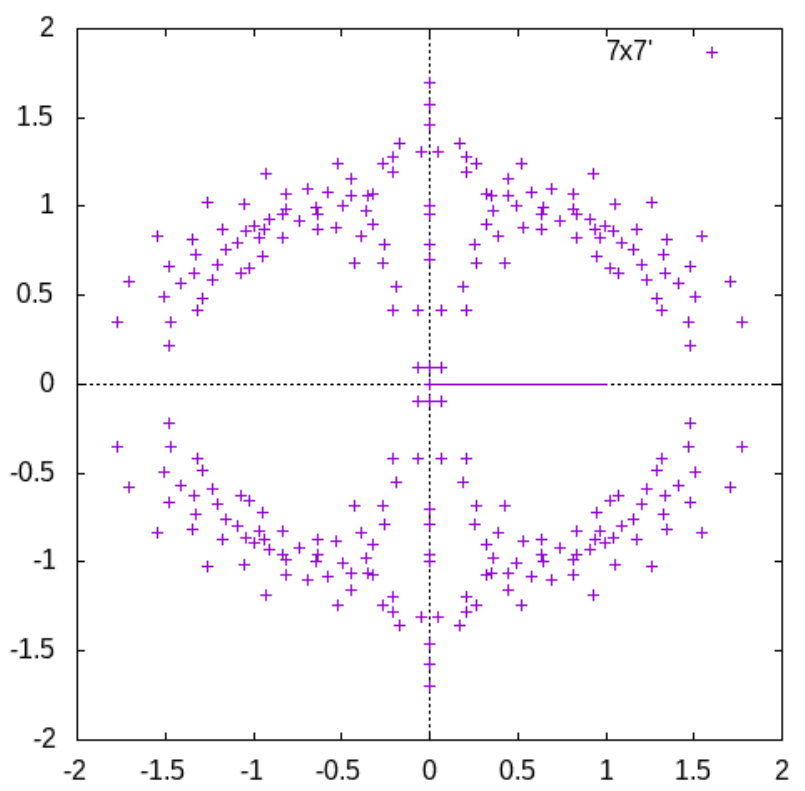

(a)

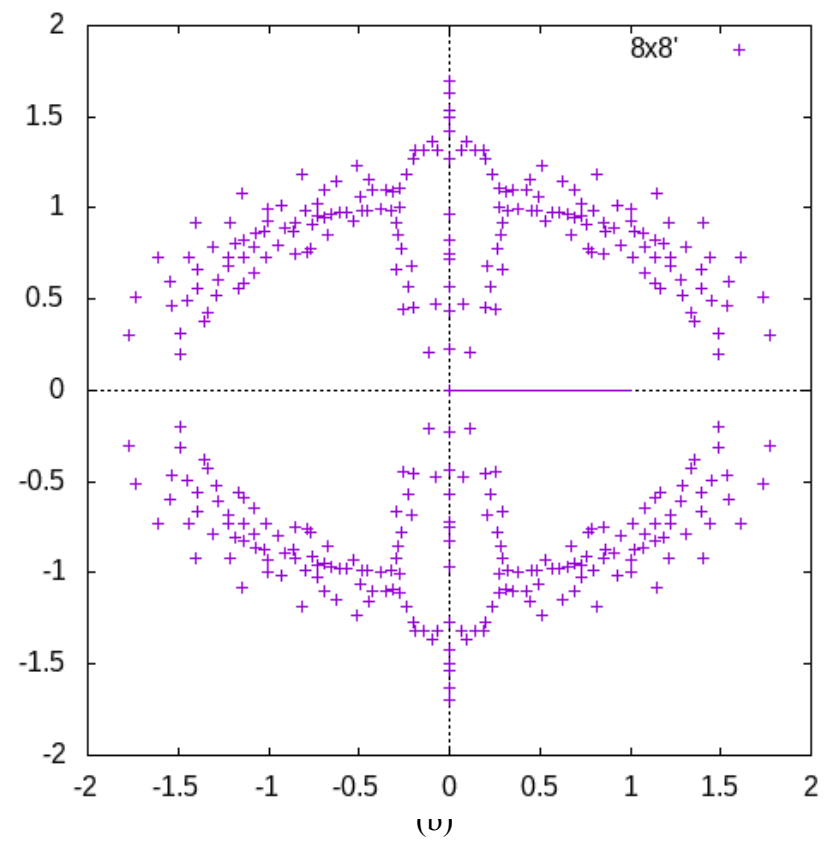

Figure 8 Zeros distribution for $\chi=(3,2,1,0)$; a) $\mathrm{N}=7$, b) $\mathrm{N}=8$.

The observation for ferromagnetic and antiferromagnetic regions is important since we can determine the behaviour of zeros approaching the real axis. The locus of zeros at thermodynamic limit for this finitely many zeros will determine the existence and number of phase transition for the physical systems. Multiple loci or arcs of zeros suggest for multiple phase transitions illustratively [1]. However, for the other regions that are unphysical, the questions regarding the pattern and locus of zeros distribution are still unknown. The physical region is interesting due to the information it gives us that is related to physical observables.

The bigger lattice is also needed in all these cases in order to see a better illustration. For further investigation of the existence of phase transition due to number of curves, this can be done by using the equation of physical observable, called specific heat [18].

\section{Energy penalty}

Based on the result, we compare with previously studied $Z_{5^{-}}$ symmetry model [3] relating the energy penalty for each of the energy level to the number of curves in the zeros distribution (refer Table 2).

Table 2 Relationship between the energy penalty and the number of curve in the zeros distribution; (a) $Z_{5}$-symmetric model, b) $Z_{6}$-symmetric model.

\begin{tabular}{ccccc}
\hline$\chi$ & Penalty & 1 curve & $\begin{array}{c}\text { Double } \\
\text { curve }\end{array}$ & Unsure \\
\hline$(2,1,0)$ & $(1,1)$ & & & $\checkmark$ \\
$(3,1,0)$ & $(2,1)$ & $\checkmark$ & & \\
$(3,2,0)$ & $(1,2)$ & & $\checkmark$ & \\
$(4,1,0)$ & $(3,1)$ & $\checkmark$ & & \\
$(4,3,0)$ & $(1,3)$ & & $\checkmark$ & \\
$(5,1,0)$ & $(4,1)$ & $\checkmark$ & & \\
$(5,3,0)$ & $(2,3)$ & & $\checkmark$ & \\
$(5,4,0)$ & $(1,4)$ & & $\checkmark$ & \\
$(6,1,0)$ & $(5,1)$ & $\checkmark$ & & \\
$(6,5,0)$ & $(1,5)$ & & $\checkmark$ & \\
\hline
\end{tabular}

(a)

\begin{tabular}{ccccc}
\hline$\chi$ & Penalty & 1 curve & $\begin{array}{c}\text { Double } \\
\text { curve }\end{array}$ & Unsure \\
\hline$(2,1,0,0)$ & $(1,1,0)$ & & & $\checkmark$ \\
$(2,1,1,0)$ & $(1,0,1)$ & $\checkmark$ & & \\
$(3,1,0,0)$ & $(2,1,0)$ & $\checkmark$ & & \\
$(3,2,0,0)$ & $(1,2,0)$ & & $\checkmark$ & \\
$(3,2,1,0)$ & $(1,1,1)$ & & & $\checkmark$ \\
\hline
\end{tabular}

(b)

Table 2 shows the number of curves approaching real axis in the ferromagnetic region of the zeros distribution for specific energy level $\chi$ and the value of its energy penalty. The small loss of energy is obtained for the first energy step (between $x_{1}$ and $x_{2}$ ) as compared to the second energy step (between $x_{2}$ and $x_{3}$ ) for $Q=5$, suggesting an existence (by illustration of the zeros distribution) of the multiple curves in the zeros distribution. This observation can be seen especially for the cases $(3,2,0),(4,3,0),(5,4,0),(6,5,0)$ where their first energy step is exactly 1 . Similarly, the possibility of multiple curves approaching real axis in ferromagnetic region is observed for the case $(5,3,0)$ and for $(3,2,0,0)$ for $Q=6$ when the second energy step is greater than the first energy step.

In contrary, when the first energy step is greater than the second energy step as in $(3,1,0),(4,1,0),(5,1,0),(6,1,0),(2,1,1,0)$ and $(3,1,0,0)$, only single curve is possible for the zeros distribution.

For the cases $(2,1,0),(2,1,0,0$,$) and (3,2,1,0)$, the number of curves in the graph is not very clear. These cases have equal value of energy loss between two different energy steps, that is between $x_{1}$ and $x_{2}$ and also between $x_{2}$ and $x_{3}$. The bigger lattice sizes are needed to see the zeros distribution - more points will be available in the bigger cases. Only 5 cases for $Q=6$ are managed to be considered as compared to many other cases for $Q=5$.

\section{Graph of specific heat}

We continue with the interpretation of our zeros result with respect to physical observable of specific heat and phase transition. A specific heat is the value of the heat required to raise the temperature by one degree Celcius. Discontinuity of the equation of the specific heat at the thermodynamic limit shows the existence of the phase transition. Refer 
$[1,16,18]$ for more explanations on specific heat and other functions of states.

From the classical thermodynamic relation, the Helmholtz free energy is given by

$$
F=\langle U\rangle-T S
$$

where $\langle U\rangle$ is an internal energy, $T$ is temperature, and $S$ is an entropy $[16,19]$. It is equivalent to the function given by

$$
F=-k_{B} T \ln (Z) \text {. }
$$

The specific heat $C_{v}$ is defined from the second derivative of logarithm of the partition function with respect to $\beta$ as (recall that $\beta=$ $\left.\frac{1}{k_{B} T}\right)$

$$
\frac{C_{V}}{k_{B}}=-\beta^{2} \frac{d^{2} \ln Z}{d \beta^{2}}=\beta^{2} \frac{d}{d \beta} U(\beta),
$$

where $U(\beta)$ represents the first derivative of the logarithm of the partition function which also known as the internal energy.

At infinite size, the phase transition exists when the graph of specific heat is discontinuous at $\beta_{c}$. This discontinuity point corresponds to the phase transition critical temperature $T_{c}$. At the thermodynamic limit, the exact critical point of phase transition occurs when the peak of the specific heat graph is discontinuous. From our result on the zeros distribution, this behaviour is already observed. To support our claim and observation, we present the graph of the specific heat for our model.

Figure 9(a) shows the graph of the specific heat for $Z_{6}$-symmetric model with five energy levels, $(2,1,0,0),(2,1,1,0),(3,1,0,0),(3,2,0,0)$ and $(3,2,1,0)$. For $(2,1,0,0),(2,1,1,0)$ and $(3,1,0,0)$, there is only one peak which corresponds to one phase transition. As the energy level increases, the peak becomes steeper. For $(3,2,0,0)$ and $(3,2,1,0)$, there are two peaks which correspond to two phase transitions. But the visual appearance of the multiple peaks for $(3,2,0,0)$ is clearer when compared to the peaks for $(3,2,1,0)$. This is possibly due to the different energy steps of the energy level.

As seen in Figure 9(b), we observe the behaviour of the peaks for $(3,2,0,0)$ as the lattice size increases $(\mathrm{N}=6,7,8)$. The peaks become steeper when the lattice size increases.

This observation supports our claim for the existence of the multiple phase transitions in this model since there are multiple peaks in the specific heat graphs which correspond to the multiple phase transitions.

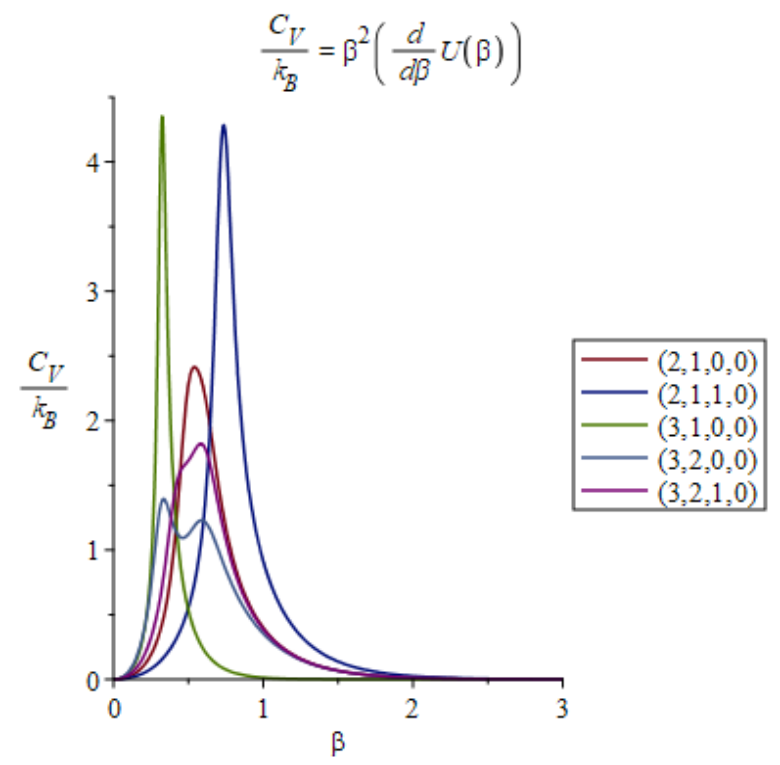

(a)

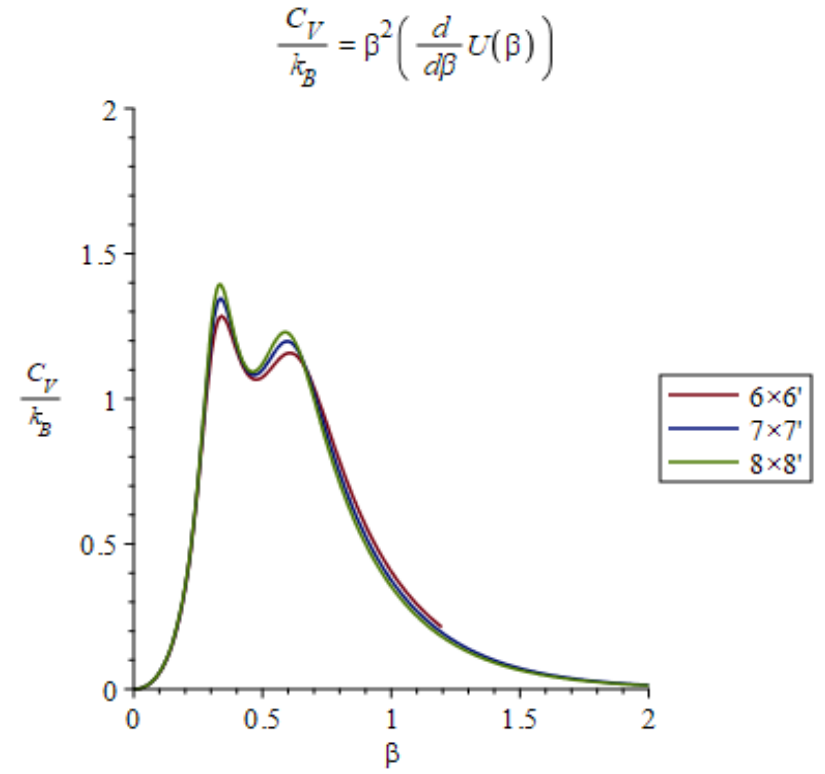

(b)

Figure 9 Graph of specific heat $C_{V}$ for (a) $8 \times 8^{\prime}$ with different $\chi$ values and (b) $6 \times 6^{\prime}, 7 \times 7^{\prime}, 8 \times 8^{\prime}$ for $\chi=(3,2,0,0)$.

\section{CONCLUSION}

We have studied the partition functions and their zeros for $Z_{6^{-}}$ symmetric models on triangular lattices. It is the extension from the study of $Z_{5}$-symmetric model. The zeros of the partition function are computed by Newton-Raphson method, and then are plotted in the complex Argand plane in order to study their distributions. From the results, the existence of multiple phase transitions is in accordance to the multiple numbers of arcs in the graph. The relation to energy penalty is also discussed relating to number of possible linear arcs. This result is supported through the observation from the peak in the specific heat graph. Our study is limited to only for some computable cases. The better computing resources (due to Moore's Law) are needed to investigate the larger lattice sizes and also for a bigger type of energy loss.

\section{ACKNOWLEDGEMENT}

This work was financially supported by the Ministry of Education Malaysia FRGS Grant, FRGS/1/2019/STG06/UIAM/03/1 (Project ID: FRGS19-122-0731).

\section{REFERENCES}

[1] P. Martin, Potts models and related problems in statistical mechanics, vol. 5, World Scientific Publishing Company, Teaneck N. J., 1991.

[2] E. Ising, Beitrag zur theorie des ferromagnetismus, Zeitschrift für Phys., vol. 31, no. 1, pp. 253-258, 1925.

[3] F. Zakaria and S. Manshur, The zeros distribution of Z 5-symmetric model on a triangular lattice, 2019. Submitted to Malaysian Journal of Fundamental and Applied Sciences.

[4] B. Kaufman, Crystal statistics. II. Partition function evaluated by Spinor analysis, Phys. Rev., vol. 76, no. 8, pp. 1232-1243, 1949.

[5] L. Onsager, "Crystal statistics. I. A two-dimensional model with an orderdisorder transition, Phys. Rev., vol. 65, no. 3-4, p. 117, 1944.

[6] R. J. Baxter, Onsager and Kaufman's calculation of the spontaneous magnetization of the Ising model: II, J. Stat. Phys., vol. 149, no. 6, pp. 1164-1167, 2012.

[7] M. E. Fisher, The nature of critical points. University of Colorado Press, 1965.

[8] F. Zakaria, Analytic properties of Potts and Ising model partition functions and the relationship between analytic properties and phase transitions in equilibrium statistical mechanics, Doctoral dissertation, University of Leeds, United Kingdom, 2016. 
[9] P. P. Martin and S. F. Zakaria, Zeros of the 3-state Potts model partition function for the square lattice revisited, J. Stat. Mech. Theory Exp. vol. 2019, no. 8, p. 84003, 2019.

[10] S. Ono, Y. Karaki, M. Suzuki, and C. Kawabata, Statistical mechanics of three-dimensional finite Ising model, Phys. Lett. A, vol. 24, no. 12, pp. 703-704, 1967.

[11] S. Katsura, Distribution of roots of the partition function in the complex temperature plane, Prog. Theor. Phys., vol. 38, no. 6, pp. 1415-1417, 1967.

[12] R. Abe, Logarithmic singularity of specific heat near the transition point in the Ising model, Prog. Theor. Phys., vol. 37, no. 6, pp. 1070-1079, 1967.

[13] R. Diestel, Graph Theory. $3^{\text {rd }}$ Edition, Springer, 2006.
[14] R. Diestel, Graph Theory, Electronic Edition 2000 (vol. 173), SpringerVerlag, New York, 2000.

[15] R. J. Baxter, Exactly Solved Models in Statistical Mechanics, Academic Press Limited, London, 1982.

[16] K. Huang, Statistical mechanics, $2^{\text {nd }}$ edition, John Wiley \& Sons Ltd, Canada, p. 493, 1987

[17] A. Papoulis and S. U. Pillai, Probability, random variables, and stochastic processes. McGraw-Hill, 2001.

[18] J. M. Yeomans, Statistical mechanics of phase transitions. Clarendon Press, United Kingdom, 1992.

[19] C.-N. Chen, C.-K. Hu, and F. Y. Wu, Partition function zeros of the square lattice Potts model, Phys. Rev. Lett., vol. 76, no. 2, p. 169, 1996. 